\title{
Neuroprotective Effects of Vitamin D in Multiple Sclerosis
}

\author{
Margaret H. Cadden', Nancy S. Koven ${ }^{1}$, Mitchell K. Ross ${ }^{2}$ \\ ${ }^{1}$ Program in Neuroscience, Bates College, Lewiston, USA; ${ }^{2}$ Department of Neurology, Central Maine Medical Center, Auburn, \\ USA. \\ Email: nkoven@bates.edu
}

Received June 28 ${ }^{\text {th }}$, 2011; revised July 25 ${ }^{\text {th }}$ 2011; accepted August $5^{\text {th }}, 2011$.

\begin{abstract}
Multiple sclerosis (MS), an auto-immune and neurodegenerative disease with no available cure, is marked by both physical and cognitive disability. In MS, central nervous system white matter lesions, believed to be consequences of inappropriate immune system reactivity, compromise inter-neuronal communication and, depending on the location of damage, beget a variety of symptoms including fatigue, loss of sensation, weakness of limbs, slowed psychomotor processing, and impaired memory. Recently, low vitamin D levels have been identified as a potential risk factor for MS, precipitating research into the immunomodulating properties of this vitamin that allow it to work in both a protective and therapeutic manner. Despite its promise as a disease-modifying agent, however, there is scant research that looks explicitly at vitamin D levels and cognitive symptoms of MS. Given the cognitive enhancing effects of vitamin D in other chronic inflammatory conditions such as chemotherapy-induced cognitive dysfunction and Alzheimer's disease, there is urgent need to research whether vitamin D may prove equally beneficial in reducing cognitive sequelae in MS. Guidelines for future research are suggested.
\end{abstract}

Keywords: Multiple Sclerosis, Vitamin D, Cognition

\section{Introduction}

Multiple sclerosis (MS) is an autoimmune, neurodegenerative disorder marked by chronic inflammation of the central nervous system (CNS). It has a complex etiology involving both genetic susceptibility and exposure to environmental factors. Although the distal cause of MS is still in question, the proximal cause of the disability is better understood. In MS patients, the immune system mistakenly learns to identify myelin, the fatty insulation surrounding axons, as 'non-self' and, thus, attacks and destroys the substance. As a consequence of reduced axonal insulation, communication between neurons is severely compromised, and long-term disturbance of neural communication may eventually lead to neural death. The combination of decreased efficacy of inter-neuronal communication and intermittent neural death is responsible for the observed symptoms of MS. The qualitative nature of MS symptoms depends upon the location of the damage and can include muscle weakness, mental and physical fatigue, loss of sensation, visual impairment, lack of coordination, muscle spasticity, bladder disturbance, sexual dysfunction, and cognitive decline [1].
MS is debilitating, moderately unpredictable, and prevalent in certain geographical locations. Although MS tends not to be life-shortening, its impact socioeconomically is second only to trauma in young adults [2]. Tracking and treatment of the disease is expensive, and, depending on the severity of the symptoms, it may be impossible for affected individuals to maintain employment [3]. It is estimated that 250,000 to 350,000 persons have a diagnosis of MS in the United States alone, with 200 new cases diagnosed every week [4]. Unfortunately, there is no cure for MS; current treatment involves regular use of disease-modifying agents (e.g., interferons) or, when needed, weekly steroid injections to depress the immune system, resulting in an increased risk for opportunistic infection throughout the body. In this paper, we will review the known immunological character of MS and then describe the possible neuroprotective effects of vitamin $\mathrm{D}$, highlighting the particular utility of vitamin D for cognitive enhancement in those affected by the illness.

\section{Immunology of MS}

MS is characterized by several inappropriate actions of 
the immune system (Figure 1). Based on genetic predisposition, individuals with MS tend to present certain human leukocyte antigen (HLA) class II receptors on their antigen presenting cells [5]. These particular HLA class II receptors are structurally more likely to expose proteins that are similar to myelin basic protein (MBP) as 'non-self' to immune system components. Genes that are associated with susceptibility on chromosome 6p21 in the area coding for the HLA are thought to account for $10 \%-60 \%$ of the genetic risk of MS [6,7]. In MS, CD4 ${ }^{+}$ T-cells become particularly activated by HLA class II receptors and, thus, become reactive against self. These auto-reactive T-cells often become further activated by an infection. The proposed mechanisms by which infection exacerbates T-cell activity is via "molecular mimicry" or via "by-stander activation" or both [5]. In 'molecular mimicry', the infectious antigen shares a similar structure to a 'self' myelin peptide and thereby activates T-cells to myelin as well as to the infectious agent. In 'bystander activation', the general immune response to the infection creates a cytokine profile that activates myelin auto-reactive T-cells, a result of the genetic profile of the HLA receptors.

The auto-reactive T-cells go on to bind to the blood brain barrier and enter the CNS through gaps created by inflammation between cerebrovascular endothelial cells. Once inside the brain and spinal cord, the T-cells react with the myelin protein surrounding the axons of neurons. This reaction creates release of inflammatory-mediating cytokines (specifically IFN- $\gamma$, IL-23, TNF- $\alpha$, LT, as well as others) and chemokines (RANTES, IP-10, IL-8,) which activate local immune cells (e.g., microglia) and recruit additional immune components to the area, including B-cells, antibodies, complement, monocytes, and mast cells [5]. A subset of the recruited B-cells become activated by the corresponding T-cells in the area and consequently releases auto-antibodies. The majority of subsequent damage is caused by the formation of an in-

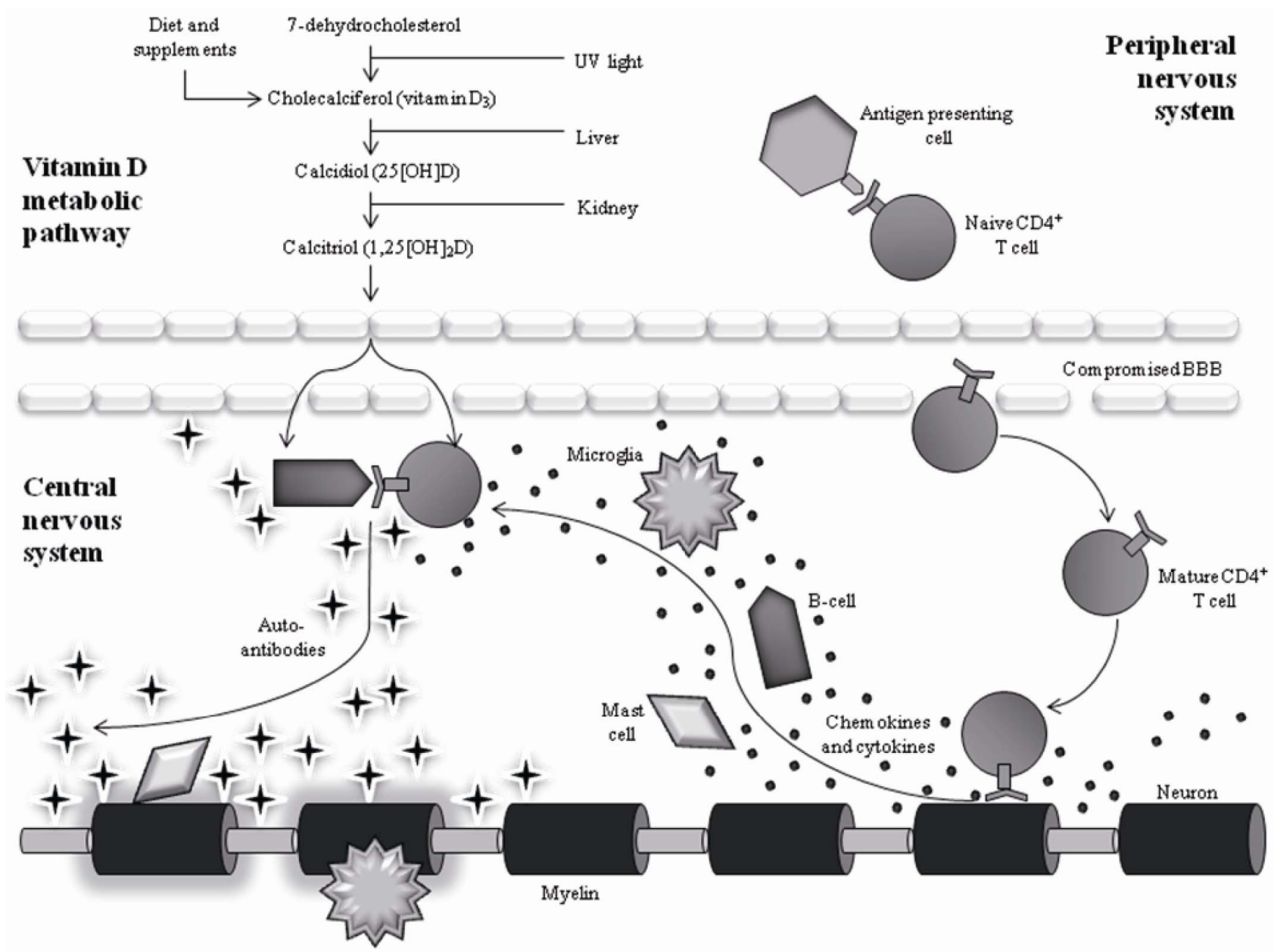

Figure 1. Interaction of Vitamin D with Immune System Components in MS. The vitamin D metabolite has the capability to interact with several immune components including B-cells, $\mathrm{CD4}^{+} \mathrm{T}$-cells, and macrophages, resulting in a decrease of cells attacking the myelin. 
flammatory lesion around the CNS tissue by the newly recruited white blood cells [5]. Formation of inflammatory lesions, typically visible on structural MRI as white matter hyperintensities, constitutes the attack or flare-up.

Although the immune-mediated destruction of myelin is thought to be the primary pathology in MS, the major cause of irreversible neurologic disability in patients is axon loss [8]. Axon loss can occur early in the disease process but is often clinically silent due to the compensatory capacity of the cortex via functional reorganization in neural networks [9]. Despite this functional compensation, advances in axon staining techniques make it clear that axon transection, or the separation of axon segments proximal and distal to an injury site, begins early in MS. The process of Wallerian degeneration, which involves degeneration of the axon distal to the point of injury, starts quickly after axon transection and is followed by swelling of the axolemma and disintegration of the axonal cytoskeleton and inner organelles. Length of time for full axon degradation is longer in the CNS than in the peripheral nervous system and depends partly on axon diameter [10]. Clearance of myelin and its debris is a protracted process, with CNS myelin persisting for a time as an empty sheath [11], so much so that an individual with MS can experience significant functional axon loss even when white matter appears unchanged with structural MRI [12]. Indeed, the best predictor of cognitive dysfunction in MS is the disease burden associated with macroscopic lesions as well as subtle abnormalities in brain tissue that otherwise appears normal with conventional neuroimaging [13], the latter of which occur both in white matter and grey matter. Although there appear to be key differences in cortical versus non-cortical lesion typology (for review, see [12]), it is evident that grey matter pathology is a significant contributor to the cognitive decline experienced by patients. The nature of this cognitive dysfunction is reviewed later in the article.

\section{Vitamin D Deficiency in MS}

Recently, low vitamin D levels have been identified as a potential risk factor for MS. Evidence from human epidemiological and experimental animal studies indicates a strong possibility that vitamin D levels influence MS disease susceptibility and disease progression. The first clue that vitamin D may have an impact on MS prevalence comes from the geographical distribution of patients with the disease. As general trends, MS prevalence increases with geographical distance from the equator [14] and develops earlier in life in individuals born in areas of low sun radiation [15]. These trends, once thought to be a result of localized outbreaks of the Epstein-Barr virus, are now considered to be a result of overall lower sun exposure.

Vitamin D is obtained through two distinct pathways into the body: diet and exposure to sunlight. Sunlight, or, more specifically, ultraviolet $\mathrm{B}$ radiation, converts a cholesterol found naturally in the skin into vitamin D (Figure 1). Consumption of foods such as fatty fish, cod liver oil, and supplements increases vitamin D levels as well. Once in the body, vitamin D is immediately converted by the liver to 25-hydroxy vitamin D $(25(\mathrm{OH}) \mathrm{D})$, which is the metabolite commonly measured in blood serum to indicate overall vitamin D levels. $25(\mathrm{OH}) \mathrm{D}$ is then further hydrolyzed by the kidneys to 1,25-dihydroxy vitamin $\mathrm{D}\left(1,25(\mathrm{OH})_{2} \mathrm{D}\right)$. Due to the fact that critical immune system components (i.e., activated T-cells, Bcells, etc.) express binding sites for $1,25(\mathrm{OH})_{2} \mathrm{D}$, it is believed that this is the functioning metabolite to alter immune system function [16]. The alteration of the immune system through this mechanism is what most likely decreases MS susceptibility and symptom intensity [17].

Seasonal variation in MS symptom presentation has been noted since a landmark study by Stamp and Round in the 1970s [18]. This finding, in conjunction with seasonal variations of $25(\mathrm{OH}) \mathrm{D}$ levels in the general population, provided groundwork for subsequent connections between vitamin D and MS. Auer and colleagues [19] found that white matter lesion load, a pathophysiological marker of MS severity, is higher in the spring and early summer months than in the fall, with the supposition that season-related low vitamin D levels lead to increased lesion load after a temporal lag. Inspired by these data, Embry et al. [20] conducted a follow-up study to look at seasonal variation of vitamin D levels in healthy individuals in the same geographic region as in the Auer et al. [19] study, comparing the healthy controls' monthly vitamin D levels to the structural MRI data of the MS patients. They found that third-order polynomial curves significantly fit both the vitamin D and sMRI data when a two month lag period was incorporated, indicating that high levels of vitamin $\mathrm{D}$ two months prior to neuroimaging predict fewer lesions in patients. The combination of these two studies strongly suggests that low vitamin D levels precipitate an increase in MS symptom severity.

Seasonal differences during neonatal development also appear to predict later development of MS. In a study examining the relationship between birth month and adulthood incidence of the disease, Salzer et al. [21] found that individuals with MS were more likely to be born in the 6-month time window between February and July than the 6-month time window between August and January. While exposure to differing levels of vitamin D during fetal development may be a possible predictive 
factor of MS for offspring, the process of pregnancy itself is an interesting lens by which to evaluate the relationship between vitamin D and MS symptom severity. As a general trend across women, pregnancy, especially in the $2^{\text {nd }}$ and $3^{\text {rd }}$ trimesters, significantly increases $1,25(\mathrm{OH})_{2} \mathrm{D}$ levels. These levels then substantially drop post-partum [22]. Frith and McLeod [23] observed that women with relapsing-remitting MS were significantly less likely to relapse during their second and third trimesters when their vitamin D levels were high, yet more likely to relapse during the three month period postpartum when their vitamin D levels were low.

Perhaps the most convincing evidence of the relationship between vitamin D and MS is found in the pilot studies in which vitamin D blood serum levels are measured in patients with MS. In general, lower vitamin $\mathrm{D}$ blood serum levels have been found in patients currently experiencing a relapse [24]. Additionally, higher levels have been shown to correlate inversely with disease exacerbation [25]. A longitudinal study tracked dietary intake as well as serum levels of vitamin $\mathrm{D}$ in American nurses [26], finding that those who reported taking a high dose of vitamin $\mathrm{D}$ generally had higher vitamin D serum levels and were consequently 40\% less likely to develop MS later in life. In recent years, a few small unblinded studies have used vitamin D supplements as therapy for MS with promising results. In the largest and only study to include a control group, Burton et al. [27] directed patients with MS to follow a varying dose protocol, which involved taking an increasing (4000 - 40,000 IU) daily dose of vitamin D over a series of 28 weeks, followed by a dose of 10,000 IU daily for the following 12 weeks, then a dose of 8000 IU daily for the 8 weeks, and finally no dose for four weeks. Results showed that members of the intervention group experienced significantly fewer relapses than those in the control group over the 52-week period.

All in all, convincing correlational evidence exists to support the idea that high vitamin D levels work in both a protective and therapeutic manner in persons with MS. Additionally, animal studies, which have far fewer confounds than human studies, have produced similar results. One study found that large $1,25(\mathrm{OH})_{2} \mathrm{D}$ administration prior to experimental autoimmune encephalomyelitis-induction (EAE, the animal model of MS) prevented animals from developing the disease [28]. This same study found that EAE mice who were fed diets high in vitamin $\mathrm{D}$ presented with fewer physical symptoms of the disease relative to non-supplemented EAE mice. Interestingly, when vitamin D was removed from the diet in the experimental condition, these mice quickly started to develop new physical MS symptoms. These results suggest that vitamin $\mathrm{D}$ works as a protector from EAE progression in mice, further strengthening the idea that vitamin $\mathrm{D}$ is working directly to prevent/alleviate MS symptoms in humans.

\section{Neuroprotective Mechanism of Vitamin D}

Although evidence to implicate vitamin $\mathrm{D}$ as a protective factor certainly exists, questions remain as to the exact mechanism by which this protection is achieved. Although there is no consensus to date in the literature, several studies have found potential answers in the immunomodulating properties of vitamin D. Key components of the immune system, including monocytes, dendritic cells, B-cells, CD4 ${ }^{+}$T-cells, and macrophages, express a subtype of the steroid-receptor super family called the vitamin D receptor [29]. Additionally, monocytes, macrophages, and T-lymphocytes increase their vitamin $\mathrm{D}$ receptors in response to $1,25(\mathrm{OH})_{2} \mathrm{D}$ exposure, indicating a possible feedback mechanism between vitamin D and immune system functioning [29] (Figure 1). Overall, it can be said that the transcription, proliferation, and differentiation of each of these immune cells are changed depending on circulating vitamin D levels in the blood [29,30]. Thus, it can be expected that varying levels of vitamin $\mathrm{D}$ will have an impact on autoimmune diseases like MS.

Supporting this notion, Correale and colleagues [16] compared 152 MS patients to control participants matched for age, gender, race, and place of residence. They looked at naturally occurring $1,25(\mathrm{OH})_{2} \mathrm{D}$ and $25(\mathrm{OH}) \mathrm{D}$ levels in both groups, as well as effects of increased vitamin $1,25(\mathrm{OH})_{2} \mathrm{D}$ levels on $\mathrm{CD}^{+}$T-cells and myelinspecific peptide T-cell lines. Levels of both $1,25(\mathrm{OH})_{2} \mathrm{D}$ and $25(\mathrm{OH}) \mathrm{D}$ were significantly lower in relapsingremitting MS patients compared to controls. Additionally, patients during the "relapse" phase of the disease had significantly lower levels of both vitamin D metabolites compared to the 'remitting' phase [16]. These researchers also found that proliferation of $\mathrm{CD} 4^{+} \mathrm{T}$-cells as well as myelin-specific peptide T-cells, both of which are involved in the learned immunity of attacking myelin, were inhibited by $1,25(\mathrm{OH})_{2} \mathrm{D}$ in a concentration-dependent manner. Interestingly, incubation of 25(OH)D with $\mathrm{CD} 4{ }^{+} \mathrm{T}$-cells resulted in an increase in $1,25(\mathrm{OH})_{2} \mathrm{D}$, and low presence of vitamin $\mathrm{D}$ receptor mRNA in $\mathrm{CD} 4^{+} \mathrm{T}$ cells increased 5-10 fold after cell activation. These results suggest that $\mathrm{CD} 4^{+} \mathrm{T}$-cells are capable of hydrolyzing $25(\mathrm{OH}) \mathrm{D}$ into $1,25(\mathrm{OH})_{2} \mathrm{D}$, which, in turn, inhibits $\mathrm{CD}^{+} \mathrm{T}$ as well as other $\mathrm{T}$-cell functioning [16]. It is possible, then, that vitamin $\mathrm{D}$ is a key component in establishing an ideal CD4 ${ }^{+} \mathrm{T}$-cell equilibrium.

There also appears to be a relationship between vitamin D and humoral immune functioning. Correale et al. [16] looked at the impact of $1,25(\mathrm{OH})_{2} \mathrm{D}$ on general 
cytokine production. More specifically, they assessed cytokine production of purified $\mathrm{CD}^{+}{ }^{+} \mathrm{T}$-cells and MBPspecific T-cells, which were pharmacologically stimulated by anti-CD3 mAb (monoclonal antibody), both in the presence and absence of vitamin D. Presence of vitamin $\mathrm{D}$ led to an increase in the number of T-cells producing IL-10 but a decrease in the number of T-cells producing IL-6 and IL-7 [16]. Since IL-6 and IL-7 cytokine profiles are associated with high inflammation, decreasing the cells that secrete these would be beneficial to prevent or alleviate inflammatory lesions such as those associated with MS.

Genetics research has also helped to identify the mechanisms by which vitamin D may be neuroprotective. For example, Simon et al. [31] identified a specific genotype of vitamin D receptor that, when present, enables vitamin $\mathrm{D}$ to act in a protective fashion. These authors suggest that, while abnormal vitamin $\mathrm{D}$ receptors may not be part of the etiology of MS, individuals with high density of vitamin $\mathrm{D}$ receptors are more likely to benefit from dietary intake of vitamin D. In another study, Fukazawa et al. [32] found that patients with MS significantly expressed more of the homozygous 'bb' profile for the vitamin $\mathrm{D}$ receptor in comparison to healthy controls. The conjunction of these two studies, although not in complete agreement as to the precise neuroprotective mechanism of vitamin $D$, further establishes an important relationship between vitamin D and MS.

\section{Vitamin $D$ as an Agent for Cognitive Enhancement}

Although there are promising data to suggest an ameliorative effect of vitamin $\mathrm{D}$ for the physical impairments of MS, it is an open question as to whether vitamin D has benefits for the cognitive sequelae of MS. The cognitive changes associated with MS are often the hardest to identify, due to the fact that they are not outwardly visible, yet tend to be the most intrusive to everyday functioning [1]. Indeed, research has shown that patients with MS may manifest cognitive impairment even when physical disability is mild [33]. Furthermore, cognitive impairment is associated with worse functional outcomes, such as decreased employment [34,35], difficulty completing activities of daily living [36], reduced participation in social and vocational activities [37] and greater rates of comorbid psychopathology [38]. With cognitive dysfunction reported by approximately $70 \%$ of patients [39], cognitive status is clearly a major variable in determining quality of life.

From the patient perspective, one of the most frequently reported complaints is decline in memory [40]. However, because memory itself is a multifaceted phenomenon, meta-analysis emerges as a useful tool to pinpo- int which aspects of memory are most affected in MS. Substantial deficits are found in both working memory, the processing of short lived, 'on-line' information, and long term memory, an unlimited permanent memory store [41]. Other research has found that long term memory deficits may be a result of a specific weakness in retrieval as opposed to encoding or storage [42]. Metaanalysis [43] reveals four additional cognitive domains that are commonly impaired in patients with MS: verbal skills, attention/concentration, cognitive flexibility and abstraction, and manual dexterity. Deficits in verbal skills typically manifest as poor scores on measures of verbal fluency (e.g., Controlled Oral Word Association Test), measures of verbal long term memory (e.g., California Verbal Learning Test), and measures of word retrieval (e.g., Boston Naming Test). Deficits in attention and concentration are evidenced by a small disparity between normal healthy controls and MS patients on the Digit Span subtest of the Wechsler Adult Intelligence Scale, Revised (WAIS-R). Larger disparities are found in tests examining information processing speed, with MS patients performing significantly worse on the Symbol Digit Modalities Test and the Paced Auditory Serial Addition Test than normal healthy controls [43]. Patients with MS are found to have moderate impairments on tests of concept formation and mental flexibility such as the Categories Test, Raven's Progressive Matrices, the Stroop Test, Trail-Making Test, the Similarities subtest of the WAIS-R, and the Wisconsin Card Sorting Test. Finally, manual dexterity and motor speed impairments in patients with MS are reflected by low performance on tests like Grooved Pegboard and Finger Tapping [43].

Pharmacological interventions for the cognitive disability associated with MS are typically disease-modifying therapies (e.g., interferons, glatiramer acetate), which likely achieve short-term effects through anti-inflammatory action and long-term effects through reduction in cerebral atrophy. Other drug interventions are symptomatic medications (e.g., acetylcholinesterase inhibitors, glutamate antagonists) which are designed as cognitive enhancers and often used in other neurological conditions such as traumatic brain injury, dementias, and attention deficit disorder. Unfortunately, neither pharmacological approach has proven reliably effective to reduce cognitive symptoms for MS patients [for review, see 44]. Although vitamin D shows promise as a diseasemodifying agent, there is scant research that looks explicitly at vitamin D levels and cognitive symptoms of MS. However, a few studies do exist that examine the relationship between vitamin $\mathrm{D}$ and cognitive symptoms of other inflammatory conditions, such as chemotherapyinduced cognitive dysfunction and Alzheimer's disease. These studies may give insight into how well vitamin $\mathrm{D}$ 
will work in attenuating the cognitive symptoms of MS.

Cancer survivors constitute an important clinical population by which to judge the merits of vitamin D administration as cognitively-enhancing, particularly those survivors who have received chemotherapy as part of their treatment regimen. Common to the $100^{+}$available chemotherapy drugs is their affinity for blocking specific pathophysiological mechanisms or components of cancerous human cells, which unfortunately can also mean blocking vital mechanisms or components of healthy cells. Recent studies have discovered greater-than-expected concentrations of chemotherapy agents in the cerebrospinal fluid and brain tissue, and it has been suggested that virtually all commonly used chemotherapy drugs are capable of permeating the blood brain barrier $[45,46]$. Three nonexclusive mechanisms are proposed to explain brain insult by these agents. Demyelination is thought to occur by direct neurotoxic assault to the cerebral parenchyma, including microglia, oligodendrocytes, and axons. Secondary inflammatory response may result from allergic hypersensitivity and autoimmune vasculitis. Third, microvascular injury caused by either direct neurotoxic assault or secondary inflammatory response may block small- to medium-sized blood vessels, instigating ischemia/infarction and parenchymal necrosis [47]. Numerous studies have offered evidence for chemotherapy-induced adverse neuropsychological sequelae, independent of the effects of depression or anxiety, in the domains of memory, mental flexibility, processing speed, attention, visuospatial ability, and motor function [48-51]. These impairments are clinically significant, with pervasive impact on functioning in the home, social, educational, and/or professional environments [52]. Although research on outcomes of vitamin supplementation on chemotherapy-induced cognitive dysfunction is scant, one important study indicates a positive role of vitamin D. In a study of women with early-stage breast cancer who were being treated with chemotherapy, Simone et al. [53] report that $88 \%$ of the sample indicated improvement in cognitive abilities after completion of a 10-point plan that included a supplement regimen high in vitamin D. Although additional research would be needed to isolate the contribution of vitamin $\mathrm{D}$ alone, these results show therapeutic promise for vitamin D in the context of chemotherapy-induced cognitive dysfunction.

More research has examined the results of vitamin D on cognition in Alzheimer's disease, again with promising results. Oudshoorn et al. [54], for example, reported that Alzheimer's patients with normal to high vitamin D blood levels perform significantly better on the MiniMental State Examination (MMSE), a common bedside assessment of cognition, relative to those with low vita- min D levels. Another study conducted with patients with mild forms of dementia found that participants with low vitamin D levels were more likely to have an active mood disorder such as depression, to score lower on a measure of memory and concentration (e.g., Short Blessed Test), and to have higher overall dementia scores [55]. There is evidence as well to suggest cognitive benefits from vitamin D in non-demented older adults. In a population-based study of 5,596 community-dwelling women (mean age $80.5 \pm 0.1$ years), Annweiler et al. [56] found that a subset of participants with high vitamin $D$ levels outperformed a subset with low levels on the Pfeiffer Short Portable Mental State Questionnaire, a measure of global cognitive functioning. Pryzbelski and Binkley [57] reported similar findings using the MMSE to assess cognition in older adults. Moreover, Llewellyn et al. [58] found that low levels of vitamin D were associated with substantial cognitive decline, as measured by scores on the MMSE and the Trail-Making Test, in 858 elderly participants who were studied prospectively over a 6-year period. Although the mechanisms that mediate these cognitive effects are not well understood, several possibilities have been suggested: increasing brain cholinergic levels via effects on choline acetyltransferase, increasing neurotrophin activity via effects on nerve growth factor synthesis, decreasing free radicals by downregulating nitric oxide and upregulating $\gamma$ glutamyl transpeptidase, and prevention of excitotoxic damage by promoting calcium homeostasis [57]. While the relative contribution of change in each of these pathways is unknown, these mechanisms are a worthwhile starting point by which to evaluate the enhancing effects of vitamin D in MS.

\section{Future Research Directions}

Taken together, researchers have amassed considerable data to suggest an important role for vitamin D in treating MS, but we still do not yet know the extent to which vitamin $\mathrm{D}$ is effective in addressing all aspects of the disease. To determine whether vitamin $\mathrm{D}$ is ameliorative for the cognitive dysfunction associated with MS, it will be imperative to utilize neuropsychological assessment measures to document intervention-specific changes. Because there is often mismatch between patterns gleaned from patient self-report measures of cognitive ability and performance-based assessments [59], neuropsychological tools have the advantage of being objective while at the same time providing opportunity for careful, qualitative observation of patient behavior during tasks. Still, self-report measures of cognition have clinical utility as ecologically valid tasks to assess broader, molar components of everyday living; we recommend that such scales be used to complement neuropsychologi- 
cal testing, particularly measures that provide self-report as well as informant-report items (e.g., the Behavior Rating Inventory of Executive Function, Adult version [60]). Given that physical and cognitive fatigue is common in MS [61] and given that comprehensive neuropsychological assessment is time-intensive, we recommend using a targeted battery of tests that is maximally sensitive to the cognitive disturbances in MS (for review, see [43]). Finally, as mood variables may affect cognitive performance [62] and in light of the relationship between vitamin D and mood [63], it is important to simultaneously assess mood functioning during neuropsychological testing. Should it appear that vitamin D does improve cognition in MS, it will be important to ascertain whether such a relationship is mediated by mood-related variables such as depression or trait anxiety.

In addition to documenting cognitive change as a result of vitamin D administration, an important research goal is to understand the pharmacological mechanisms underlying such change. With additional neuroimaging research tools, we will be able to probe changes in brain volume, functional connectivity, and neurochemical activity that are contemporaneous with behavioral changes in patient groups. High-resolution structural neuroimaging methods, which include structural magnetic resonance imaging (MRI) and diffusion tensor imaging (DTI), will permit localization of intervention-related alterations in gray and white matter on a voxel-by-voxel basis. Analysis of brain activation during presentation of experimental stimuli, including neuropsychological protocols that are compatible with functional imaging environments, is possible with various technologies, some of which are available in outpatient research settings (e.g., electroencephalography to evaluate event-related potentials; optical imaging) and some of which involve hospital-based equipment (e.g., functional MRI; magnetoencephalography; single photon emission computed tomography, SPECT; positron emission tomography, PET). Finally, with SPECT and PET, detection of neurochemical changes in the brain is possible with radioactive labeling of agents that uniquely bind to neurotransmitter receptors and transporters at the synapse. To the extent that any behavioral and physiological changes are governed by individual differences in genotypes, genetic profiling is recommended as another useful methodological approach to understand the mechanics of vitamin D-related improvement. Candidate genes are those involved in vitamin D metabolism, transport, or binding and activity at vitamin $\mathrm{D}$ receptors. Some research has already explored relationships between vitamin D-related polymorphisms and physical symptoms of MS [31,64], but no studies to date have explored connections between genotypes and cognitive phenotypes.

\section{Conclusions}

That vitamin D is important for normal brain function is supported by presence of vitamin $\mathrm{D}$ metabolites and vitamin D receptors in the central nervous system. There is overwhelming evidence, both in human and nonhuman animal studies, that highlights an association between inadequate levels of vitamin $\mathrm{D}$ and poor performance on physical and cognitive outcome measures in MS patients. The immunological mechanisms that operate in MS and the known immunomodulating properties of vitamin $\mathrm{D}$, together, provide strong rationale for continued excitement about the disease-modifying potential of vitamin D. Enhanced neuroprotection, as evidenced by positive outcomes with vitamin D administration in individuals with Alzheimer's disease and chemotherapyrelated cognitive dysfunction, could contribute to promotion and maintenance of normal cognition. Future research is clearly needed; neuropsychological assessment, in conjunction with physiological and genetic methodologies, is a tripartite approach by which to study the promise of vitamin D as therapeutic for MS. Pending the outcomes of such research, vitamin D may very well be indicated as an accessible and inexpensive supplement to MS treatment regimens.

\section{REFERENCES}

[1] R. G. Frank and T. R. Elliot, "Handbook of Rehabilitation Psychology," American Psychological Association, Washington, D.C., 2000. doi:10.1037/10361-000

[2] D. E. McFarlin and H. F. McFarland, "Multiple Sclerosis (First of Two Parts)," New England Journal of Medicine, Vol. 307, No. 19, 1982, pp. 1183-1188. doi:10.1056/NEJM198211043071905

[3] Canadian Burden of Illness Study Group, "Burden of Illness of Multiple Sclerosis: Part 1. Cost of Illness,” $\mathrm{Ca}$ nadian Journal of Neurological Sciences, Vol. 25, 1988, pp. 23-30.

[4] National Institute of Health, "NINDS Multiple Sclerosis Information Page,” 2010. http://www.nids.nih.gov/disor- ders/multiple_sclerosis.

[5] M. Sospedra and R. Martin, "Immunology of Multiple Sclerosis,” Annual Review of Immunology, Vol. 23, 2005, pp. 683-747. doi:10.1146/annurev.immunol.23.021704.115707

[6] J. L. Haines, H. A. Terwedow, K. Burgess, M. A. Pericak-Vance, J. B. Rimmler, E. R. Martin, J. R. Okenberg, et al., "Linkage of the MHC to Familial Multiple Sclerosis Suggests Genetic Heterogeneity,” Human Molecular Genetics, Vol. 7, No. 8, 1998, pp. 1229-1234. doi:10.1093/hmg/7.8.1229

[7] J. Hillert and O. Olerup, "HLA and MS,” Neurology, Vol. 43, 1993, pp. 2426-2427.

[8] C. Bjartmar, J. R. Wujek and B. D. Trapp, “Axonal Loss 
in the Pathology of MS: Consequences for Understanding the Progressive Phase of the Disease," Journal of the Neurological Sciences, Vol. 206, No. 2, 2003, pp. 165-171. doi:10.1016/S0022-510X(02)00069-2

[9] A. M. Parry, R. B. Scott, J. Palace, S. Smith and P. M. Matthews, "Potentially Adaptive Functional Changes in Cognitive Processing for Patients with Multiple Sclerosis and Their Acute Modulation by Rivastigmine," Brain, Vol. 126, No. 12, 2003, pp. 2750-2760. doi:10.1093/brain/awg284

[10] M. Kerschensteiner, M. E. Schwab, J. W. Lichtman and T. Misgeld, "In Vivo Imaging of Axonal Degeneration and Regeneration in the Injured Spinal Cord,” Nature Medicine, Vol. 11, No. 5, 2005, pp. 572-577. doi:10.1038/nm1229

[11] C. Bjartmar, G. Kidd, S. Mork, R. Rudick and B. D. Trapp, "Neurological Disability Correlates with Spinal Cord Axonal Loss and Reduced N-acetyl Aspartate in Chronic Multiple Sclerosis Patients," Annals of Neurology, Vol. 48, No. 6, 2000, pp. 893-901.

doi:10.1002/1531-8249(200012)48:6<893::AID-ANA10> 3.0.CO;2-B

[12] R. Dutta and B. D. Trapp, "Pathogenesis of Axonal and Neuronal Damage in Multiple Sclerosis,” Neurology, Vol. 68, Suppl. 3, 2007, pp. S22-S31. doi:10.1212/01.wnl.0000275229.13012.32

[13] M. Filippi, C. Tortorella, M. Rovaris, M. Bozzali, F. Possa, M.P. Sormani, et al., "Changes in the Normal Appearing Brain Tissue and Cognitive Impairment in Multiple Sclerosis," Journal of Neurology, Neurosurgery, and Psychiatry, Vol. 68, No. 2, 2000, pp. 157-161. doi:10.1136/jnnp.68.2.157

[14] K. M. Myhr, "Vitamin D Treatment in Multiple Sclerosis,” Journal of Neurological Sciences, Vol. 286, No. 1-2, 2009, pp. 104-108. doi:10.1016/j.jns.2009.05.002

[15] T. Y. McDowell, S. Amr, P. Langenberg, W. Royal and C. Bever, "Time of Birth, Residential Solar Radiation, and Age at Onset of Multiple Sclerosis," Neuroepidemiology, Vol. 34, No. 4, 2010, pp. 238-244. doi:10.1159/000297749

[16] J. Correale, M. C. Ysrraelit and M. I. Gaitán, "Immunomodulatory Effects of Vitamin D in Multiple Sclerosis," Brain, Vol. 132, 2009, pp. 1146-1160. doi:10.1093/brain/awp033

[17] J. Smolders, J. Damoiseaux, P. Menheere and R. Hupperts, "Vitamin D as an Immune Modulator in Multiple Sclerosis: A Review," Journal of Neuroimmunology, Vol. 194, No. 1-2, 2008, pp. 7-17. doi:10.1016/j.jneuroim.2007.11.014

[18] T. C. Stamp and J. M. Round, "Seasonal Changes in Human Plasma Levels of 25 Hydroxyvitamin D,” Nature, Vol. 247, No. 442, 1974, pp. 563-565. doi:10.1038/247563a0

[19] D. P. Auer, E. M. Schumann, T. Kumpfel, C. Gossl and C. Trenkwalder, "Seasonal Fluctuations of Gadolinium-Enhancing Magnetic Resonance Imaging Lesions in Multiple Sclerosis,” Annals of Neurology, Vol. 47, 2000, pp.
276-277.

doi:10.1002/1531-8249(200002)47:2<276::AID-ANA28> 3.0.CO;2-1

[20] A. F. Embry, L. R. Snowdon and R. Veith, "Vitamin D and Seasonal Fluctuations of Gadolinium-Enhancing Magnetic Resonance Imaging Lesions in Multiple Sclerosis," Annals of Neurology, Vol. 48, No. 2, 2000, pp. 271272.

doi:10.1002/1531-8249(200008)48:2<271::AID-ANA28> 3.0.CO;2-O

[21] J. Salzer, A. Svenningson and P. Sundstorm, "Season of Birth and Multiple Sclerosis in Sweden," Acta Neurologica Scandinavica, Vol. 121, No. 1, 2010, pp. 20-23. doi:10.1111/j.1600-0404.2009.01181.x

[22] J. Verhaeghe and R. Bouillon, "Calciotropic Hormones during Reproduction,” Journal of Steroid Biochemistry and Molecular Biology, Vol. 41, No. 3-8, 1992, pp. 469-477. doi:10.1016/0960-0760(92)90372-P

[23] J. A. Frith and J. G. McLeod, "Pregnancy and Multiple Sclerosis," Journal of Neurology, Neurosurgery, and Psychiatry, Vol. 51, 1998, pp. 495-498. doi:10.1136/jnnp.51.4.495

[24] M. Soilu-Hanninen, M. Laaksonen, I. Laitinen, J. P. Eralinna, E. M. Lilius and I. A. Mononen, "Longitudinal Study of Serum 25-Hydroxyvitamin D and Intact PTH Levels Indicate the Importance of Vitamin D and Calcium Homeostasis Regulation in Multiple Sclerosis," Journal of Neurology, Neurosurgery, and Psychiatry, Vol. 79, No. 2, 2008, pp. 152-157. doi:10.1136/jnnp.2006.105320

[25] K. L. Munger, S. M. Zhang, E. O’Reilly, M. A. Hernán, M. J. Olek, W. C. Willett and A. Ascherio, "Vitamin D In- take and Incidence of Multiple Sclerosis,” Neurology, Vol. 62, No. 1, 2004, pp. 60-65.

[26] K. L. Munger, L. I. Levin, B. W. Hollis, N. S. Howards and A. Ascherio, "Serum 25-Hydroxyvitamin D Levels and Risk of Multiple Sclerosis,” Journal of the American Medical Association, Vol. 296, No. 23, 2006, pp. 2832 2838. doi:10.1001/jama.296.23. 2832

[27] J. M. Burton, S. M. Kimball, R. Veith, A. Bar-Or, H. M. Dosch, L. Thibault, et al., "A Phase I/II Dose Escalation Trial of Oral Vitamin D3 with Calcium Supplementation in Patients with Multiple Sclerosis," Multiple Sclerosis, Vol. 14, Suppl. 1, 2008, p. 34.

[28] M. T. Cantorna, C. E. Hayes and H. F. DeLuca, “1,25Hydroxyvitamin D3 Reversibly Blocks the Progression of Relapsing Encephalomyelitis: A Model of Multiple Sclerosis," Proceedings of the National Academy of Sciences of the United States of America, Vol. 93, No. 15, 1996, pp. 7861-7864. doi:10.1073/pnas.93.15.7861

[29] C. M. Veldman, M. T. Cantorna and H. F. DeLuca, "Expression of $1,25(\mathrm{OH})_{2}$ D3 Receptors in the Immune System,” Biochemical Biophysiology, Vol. 374, No. 2, 2000, pp. 334-339. doi:10.1006/abbi.1999.1605

[30] D. M. Provvendini, C. D. Tsoukas, L. J. Deftos and S. C. Manolagas, "1,25(OH) $)_{2}$ D3 Receptors in Human Leukocytes,” Science, Vol. 221, No. 4616, 1983, pp. 1181-1183. doi:10.1126/science.6310748 
[31] K.C. Simon, K.L. Munger, L. Xing and A. Ascherio, "Polymorphisms in Vitamin D Metabolism Related Genes and Risk of Multiple Sclerosis," Multiple Sclerosis, Vol. 16, No. 2, 2010, pp. 133-138. doi:10.1177/1352458509355069

[32] T. Fukazawa, I. Yabe, S. Kikuchi, H. Sasaki, T. Hamada, K. Miyasaka, et al., "Association of Vitamin D Receptor Gene Polymorphism with Multiple Sclerosis in Japanese," Journal of the Neurological Sciences, Vol. 166, 1999, pp. 47-52. doi:10.1016/ S0022-510X(99)00112-4

[33] R. M. Ruggieri, R. Palermo, G. Vitello, M. Gennuso, N. Settipani and F. Piccoli, "Cognitive Impairment in Patients Suffering from Relapsing-Remitting Multiple Sclerosis with EDSS < or = 3.5," Acta Neurologica Scandinavica, Vol. 108, No. 5, 2003, pp. 323-326. doi:10.1034/j.1600-0404.2003.00157.x

[34] W. W. Beatty, C. R. Blanco, S. L. Wilbanks, R. H. Paul and K. A. Hames, "Demographic, Clinical, and Cognitive Characteristics of Multiple Sclerosis Patients Who Continue to Work," Journal of Neurologic Rehabilitation, Vol. 9, No. 3, 1995, pp. 167-173. doi:10.1177/154596839500900306

[35] S. A. Morrow, A. Drake, R. Zivadinov, F. Munschauer, B. Weinstock-Guttman and R. H. B. Benedict, "Predicting Loss of Employment over Three Years in Multiple Sclerosis: Clinically Meaningful Cognitive Decline," The Clinical Neuropsychologist, Vol. 24, No. 7, 2010, pp. 1131-1145. doi:10.1080/ 13854046.2010.511272

[36] H. R. Kessler, R. A. Cohen, K. Lauer and D. F. Kausch, "The Relationship between Disability and Memory Dysfunction in Multiple Sclerosis," International Journal of Neuroscience, Vol. 62, No. 1-2, 1992, pp. 17-34. doi:10.3109/00207459108999754

[37] E. A. Hakim, A. M. Bakheit, T. N. Bryant, M. W. Roberts, S. A. McIntosh-Michaelis, A. J. Spackman, et al., "The Social Impact of Multiple Sclerosis-A Study of $305 \mathrm{~Pa}-$ tients and Their Relatives," Disability and Rehabilitation, Vol. 22, No. 6, 2000, pp. 288-293. doi:10.1080/096382800296755

[38] S. M. Rao, G. J. Leo, L. Ellington, T. Nauertz, L. Bernardin and F. Unverzagt, "Cognitive Dysfunction in Multiple Sclerosis. II. Impact on Employment and Social Functioning,” Neurology, Vol. 41, No. 5, 1991, pp. 692-696.

[39] S. M. Rao, "Neuropsychological Aspects of Multiple Sclerosis,” In: C. S. Raine, H. F. McFarland and W. W. Tourtellote, Eds., Multiple Sclerosis: Clinical and Pathogenic Basis, Chapman \& Hall, London, 1997, pp. 357362.

[40] J. M. Bruce and P. A. Arnett, "Self-Reported Everyday Memory and Depression in Patients with Multiple Sclerosis," Journal of Clinical and Experimental Neuropsychology, Vol. 26, No. 2, 2004, pp. 200-214. doi:10.1076/jcen.26.2.200.28081

[41] A. E. Thornton and N. Raz, "Memory Impairment in Multiple Sclerosis: A Quantitative Review,” Neuropsychology, Vol. 11, No. 3, 1997, pp. 357-366. doi:10.1037/0894-4105.11.3.357
[42] S. M. Rao, "Neuropsychology of Multiple Sclerosis: A Critical Review," Journal of Clinical and Experimental Neuropsychology, Vol. 8, No. 5, 1986, pp. 503-542. doi:10.1080/01688638608405173

[43] K. K. Zakzanis, "Distinct Neurocognitive Profiles in Multiple Sclerosis Subtypes," Archives of Clinical Neuropsychology, Vol. 15, No. 2, 2000, pp. 115-136.

[44] F. Patti, C. Leone and E. D’Amico, "Treatment Options of Cognitive Impairment in Multiple Sclerosis,” Neurological Science, Vol. 31, Suppl. 2, 2010, pp. S265-S269. doi:10.1007/s10072-010-0438-7

[45] M.K. Tuxen and S.W. Hansen, "Neurotoxicity Secondary to Antineoplastic Drugs," Cancer Treatment Reviews, Vol. 20, No. 2, 1994, pp. 191-214. doi:10.1016/0305-7372(94)90027-2

[46] L. Troy, K. McFarland, S. Littman-Power, B. J. Kelly, E. T. Walpole, D. Wyld, et al., "Cisplatin-Based Therapy: A Neurological and Neuropsychological Review," PsychoOncology, Vol. 9, No. 1, 2000, pp. 29-39. doi:10.1002/(SICI)1099-1611(200001/02)9:1<29::AID-P ON428>3.0.CO;2-Z

[47] A. J. Saykin, T. A. Ahles and B. C. McDonald, "Mechanisms of Chemotherapy-Induced Cognitive Disorders: Neuropsychological, Pathophysiological, and Neuroimaging Perspectives," Seminars in Clinical Neuropsychiatry, Vol. 8, 2003, pp. 201-216.

[48] B. Collins, J. Mackenzie, A. Stewart, C. Bielajew and S. Verma, "Cognitive Effects of Chemotherapy in PostMenopausal Breast Cancer Patients 1 Year after Treatment," Psycho-Oncology, Vol. 18, No. 2, 2009, pp. 134143. doi:10.1002/pon.1379

[49] H. S. Jim, K. A. Donovan, B. J. Small, M. A. Andrykowski, P. N. Munster and P. B. Jacobsen, "Cognitive Functioning in Breast Cancer Survivors: A Controlled Comparison,” Cancer, Vol. 115, No. 8, 2009, pp. 1177611783. doi:10.1002/cncr.24192

[50] J. Vardy, J. S. Wefel, T. Ahles, I. F. Tannock and S. B. Schagen, "Cancer and Cancer-Therapy Related Cognitive Dysfunction: An International Perspective from the Venice Cognitive Workshop," Annals of Oncology, Vol. 19, No. 4, 2008, pp. 623-629. doi:10.1093/annonc/mdm500

[51] M. H. Weineke and E. R. Dienst, "Neuropsychological Assessment of Cognitive Functioning Following Chemotherapy for Breast Cancer,” Psycho-Oncology, Vol. 4, No. 1, 1995, pp. 61-66. doi:10.1002/pon.2960040108

[52] L. A. Ouimet, A. Stewart, B. Collins, D. Schindler and C. Bielajew, "Measuring Neuropsychological Change Following Breast Cancer Treatment: An Analysis of Statistical Models," Journal of Clinical and Experimental Neuropsychology, Vol. 31, 2009, pp. 73-89. doi:10.1080/13803390801992725

[53] C. B. Simone, N. L. Simone and C. B. Simone II, "Nutritional and Lifestyle Modification to Augment Oncology Care: An overview," Journal of Orthomolecular Medicine, Vol. 12, No. 4, 1997, pp. 197-206.

[54] C. Oudshoorn, F. U. S. Mattace-Raso, N. van der Velde, E. M. Colin and T. J. M. van der Cammen, "Higher Se- 
rum Vitamin D3 Levels are Associated with Better Cognitive Test Performance in Patients with Alzheimer's Disease," Dementia and Geriatric Cognitive Disorders, Vol. 25, No. 6, 2008, pp. 539-543. doi:10.1159/000134382

[55] C. H. Wilkins, Y. I. Sheline, C. M. Roe, S. J. Birge and J. C. Morris, "Vitamin D Deficiency Is Associated with Low Mood and Worse Cognitive Performance in Older Adults," American Journal of Geriatric Psychiatry, Vol. 14, No. 12, 2006, pp. 1032-1040. doi:10.1097/01.JGP.0000240986.74642.7c

[56] C. Annweiler, A. M. Schott, Y. Rolland, H. Blain, F. R. Hermann and O. Beauchet, "Dietary Intake of Vitamin D and Cognition in Older Women: A Large PopulationBased Study," Neurology, Vol. 75, No. 20, 2010, pp. 1810-1816. doi:10.1212/WNL. 0b013e3181fd6352

[57] R. J. Przybelski and N. C. Binkley, "Is Vitamin D Important for Preserving Cognition? A Positive Correlation of Serum 25-Hydroxyvitamin D Concentration with Cognitive Function," Archives of Biochemistry and Biophysics, Vol. 460, No. 2, 2007, pp. 202-205. doi:10.1016/j.abb.2006.12.018

[58] D. J. Llewellyn, I. A. Lang, K. M. Langa, G. Muniz-Terrera, C. L. Phillips, A. Cherubini, et al., "Vitamin D and Risk of Cognitive Decline in Elderly Persons," Archives of Internal Medicine, Vol. 170, No. 13, 2010, pp. 1135-1141.

doi:10.1001/archinternmed.2010.173
[59] S. Moritz, S. Ferahli and D. Naber, "Memory and Attention Performance in Psychiatric Patients: Lack of Correspondence between Clinician-Rated and Patient-Rated Functioning with Neuropsychological Test Results," Journal of the International Neuropsychological Society, Vol. 10, 2004, pp. 623-633. doi:10.1017/S1355617704104153

[60] R. M. Roth, P. K. Isquith and G. A. Gioia, "Behavior Rating Inventory of Executive Function-Adult Version," Psychological Assessment Resources, Lutz, 2005.

[61] R. H. Paul, W. W. Beatty, R. Schneider, C. R. Blanco and K. A. Hames, "Cognitive and Physical Fatigue in Multiple Sclerosis: Relations between Self-Report and Objective Performance," Applied Neuropsychology, Vol. 5, No. 3, 1998, pp. 143-148.doi:10.1207/s15324826an0503 5

[62] A. H. Kizilbash, R. D. Vanderploeg and G. Curtiss, "The Effects of Depression and Anxiety on Memory Performance," Archives of Clinical Neuropsychology, Vol. 17, No. 1, 2002, pp. 57-67.

[63] S. N. Young, "Has the Time Come for Clinical Trials on the Antidepressant Effects of Vitamin D?” Journal of Psychiatry and Neuroscience, Vol. 34, No. 1, 2009, p. 3.

[64] J. L. Steckley, D. A. Dyment, A. D. Sadovnick, N. Risch, C. Hayes and G. C. Ebers, "Genetic Analysis of Vitamin D Related Genes in Canadian Multiple Sclerosis Patients," Neurology, Vol. 54, No. 3, 2000, pp. 729-732. 